\title{
(1) Preparing the critically ill adult MICAS patient for interhospital transfer
}

Mobile Intensive Care

Ambulance Service

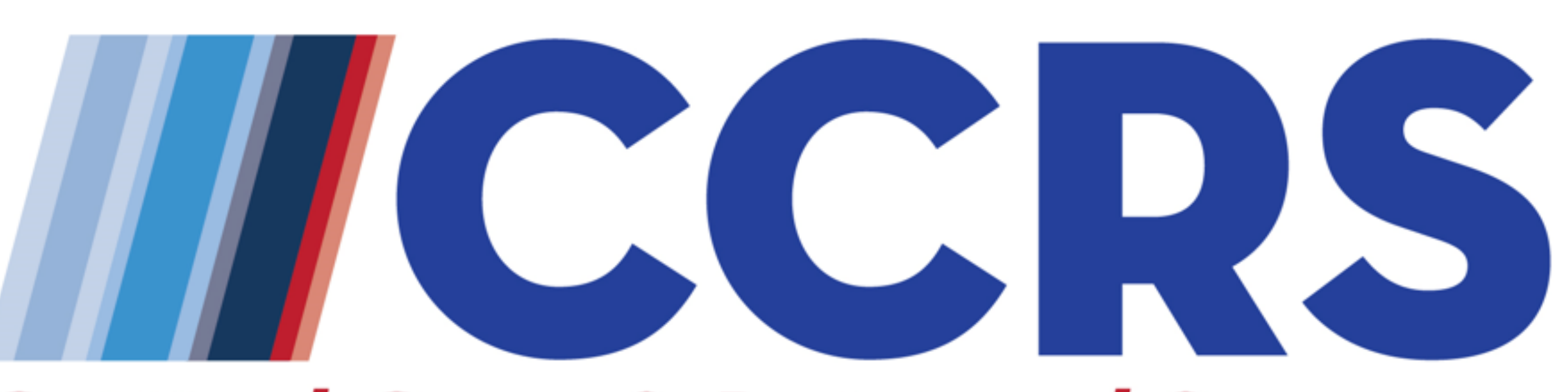

Critical Care \& Retrieval Services
David Menzies ${ }^{1}$, Anna Marie Murphy¹,

NAS Critical Care \& Retrieval Services

\section{Introduction}

The reorganisation of health services and the delivery of the 'hub and spoke' Model of Care for Critical Care necessitates transfer of critically ill patients between hospitals. In Ireland, in excess of 1000 adult and 450 paediatric critical care inter hospital transfers occur per annum. Transporting critically ill patients is associated with increased mortality and morbidity. Risk may be minimised by adequate planning, appropriate teams, training and task appropriate equipment. and there is evidence that an organised approach to transfer reduces clinical incidents.

\section{Aims}

To introduce a systematic approach to patient management when transporting critically ill patients, which establishes a higher level of performance, reduces cognitive dissonance, provides a framework for clinical teams and reduces the potential for human error.

\section{Equipment}

Rationalise medications and infusions to only those necessary during transport. All equipment secured to critical care transport trolley, ideally below the patient. Adequate battery and oxygen supplies for the journey with a $100 \%$ safety factor.

\section{Packaging}

- Pack patient in layers:

- "Dirty" lower layer - catheter, drains, ECG leads.

- "Clean" upper layer - infusions, arterial line

- Ventilator tubing external.

- Vacuum mattress as routine, even for non spinal injured patients.

SpO2 probe and IV access accessible

\section{Conclusions}

A systematic approach to preparation for transporting critically ill patients establishes a common model of care for these patients nationally. This method has been taught on over 50 outreach courses to date for hospital and NAS staff.

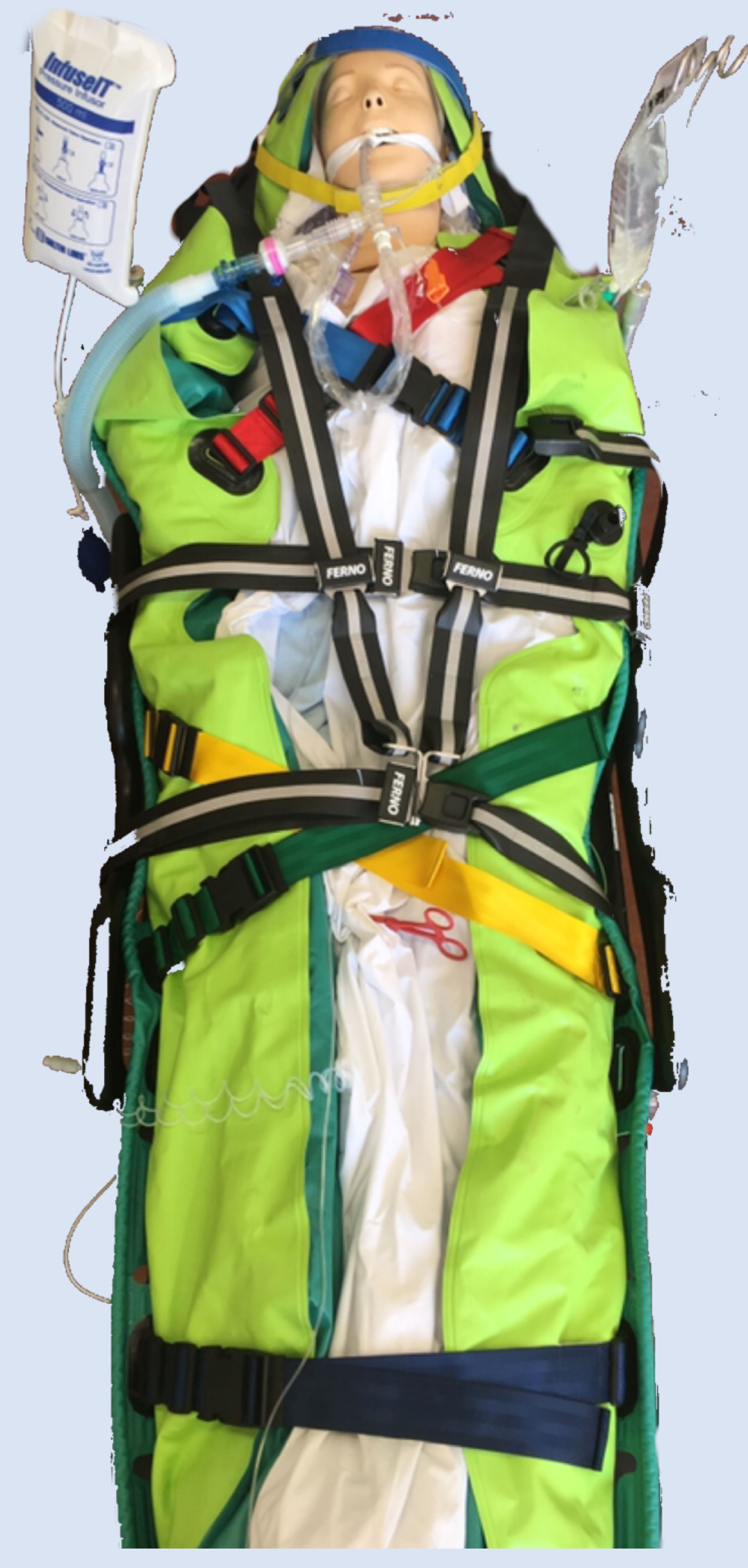

References

1. Murphy, A.M. and Dwyer, R. (2016) 'National Survey of Adult Inter-hospital transfers in Ireland' Intensive Care Society of Ireland, $11^{\text {th }}$ June.

2. AAGBI (2009) Inter hospital Transfer 3. Fried, M. et al (2010) Inter-hospital transfers of acutely ill adults in Scotland, Anaesthesia

4. National Institute for Health and Care Excellence (2017) Standardised systems of care for intra and inter hospital transfers

5. Droogh, JM., Smit, M., Hut, J.,

Absalom, A.R. Ligtenberg, JJ. and Zijlstra

JG. (2015) 'Transferring the critically ill

patient: are we there yet?, Critical Care, $19(1)$

\section{Patient Assessment}

Systematic assessment of patient condition, physiology, supports and medications using a standardised checklist.

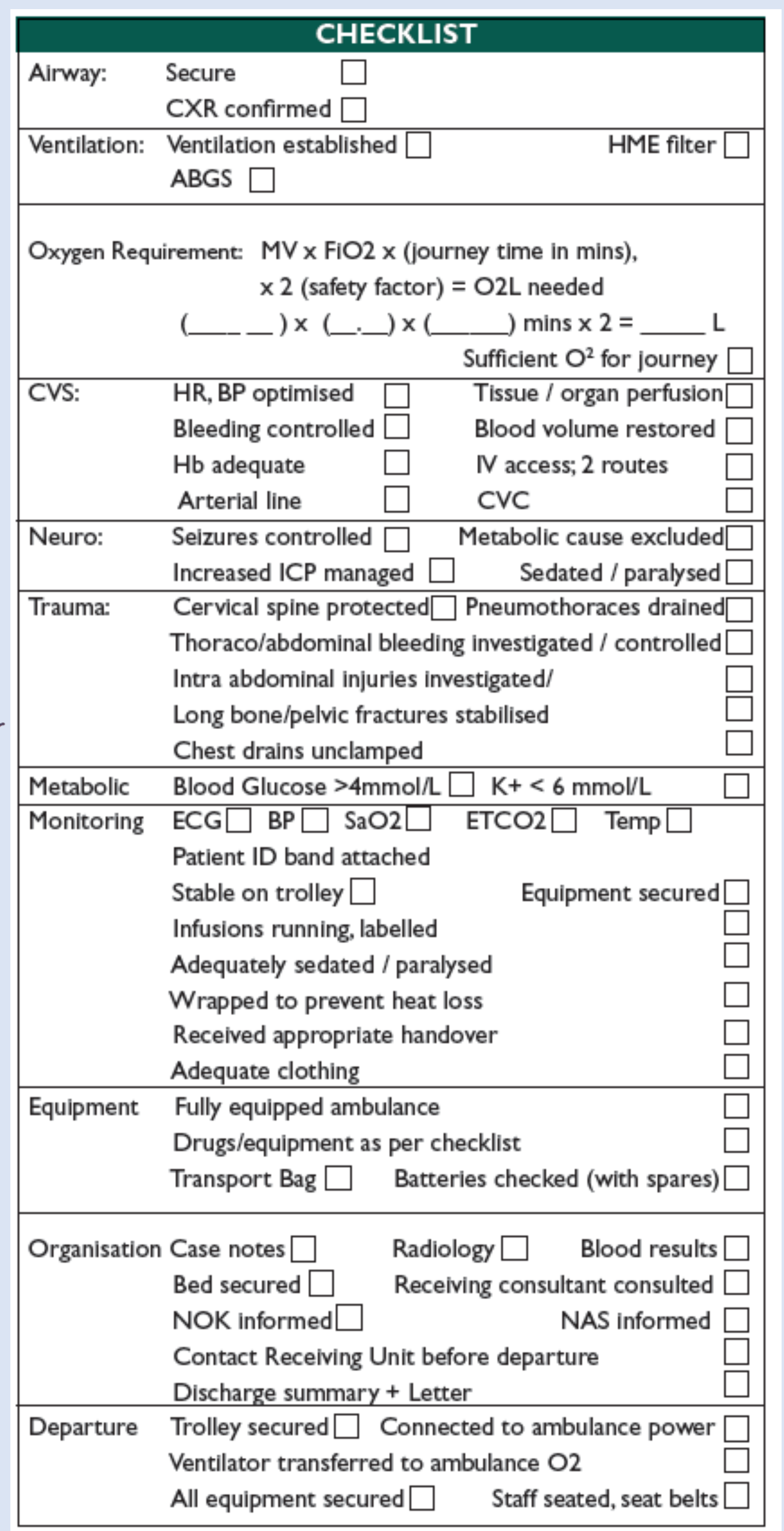

